Case Report

\title{
Isolated Cardiac Sarcoidosis with High-Grade Heart Block: Utilization of New Diagnostic Guidelines
}

\author{
Matthew R. Petersen $\mathbb{D}^{1},{ }^{1}$ Christopher Perry $\mathbb{D}{ }^{2}$ and Rachel Nickels $\mathbb{D}^{3}$ \\ ${ }^{1}$ Department of Internal Medicine, University of Florida, USA \\ ${ }^{2}$ Department of Cardiology, University of Florida, USA \\ ${ }^{3}$ United States Department of Veterans Affairs, North Florida South Georgia VA Hospital, USA \\ Correspondence should be addressed to Matthew R. Petersen; matthew.petersen@medicine.ufl.edu
}

Received 26 March 2021; Accepted 5 July 2021; Published 29 July 2021

Academic Editor: Ertugrul Ercan

Copyright (C) 2021 Matthew R. Petersen et al. This is an open access article distributed under the Creative Commons Attribution License, which permits unrestricted use, distribution, and reproduction in any medium, provided the original work is properly cited.

Cardiac sarcoidosis can present with heart failure and conduction disease. This is a case of a 58-year-old male who presented for dyspnea, edema, and varying degrees of heart block. Using new updated diagnostic guidelines and multimodal cardiac imaging, he was diagnosed with isolated cardiac sarcoidosis.

\section{History of Present Illness}

A 58-year-old male presented to the emergency room with bradycardia and lower extremity edema. A few weeks prior, he originally presented to a neighboring hospital for new onset dyspnea on exertion and lower extremity edema. On arrival, ECG showed baseline atrial fibrillation with junctional escape rhythm at a rate of 35-40 beats per minute. $\mathrm{He}$ was normotensive, and his only complaints were lower extremity edema and subacute fatigue.

Physical exam revealed an obese middle-aged male in no acute distress. His was mildly tachypneic, was sitting upright on the edge of the bed, and his lung exam revealed bibasilar crackles. His heart rate was slowed but was in a regular rhythm. He had 2+ bilateral lower extremity edema, and his extremities were warm. Initial labs revealed hemoglobin of $13.4 \mathrm{~g} / \mathrm{dL}$, hematocrit $42.2 \%$, and platelets $143 \mathrm{k} / \mathrm{cmm}$ with normal differential. Metabolic panel revealed electrolytes within normal limits; serum creatinine was $1.4 \mathrm{mg} / \mathrm{dL}$ which was slightly above the patients baseline of $1.2 \mathrm{mg} / \mathrm{dL}$. Albumin, calcium, transaminases, and bilirubin were within normal limits. High sensitivity troponin was $32 \mathrm{ng} / \mathrm{L}$ with no change on serial repeat tests, and NT pro BNP was $3018 \mathrm{pg} / \mathrm{mL}$.

\section{Past Medical History}

The patient had a past medical history of obesity and asymptomatic bradycardia first reported several years prior to presentation. He recently had been diagnosed with heart failure with preserved ejection fraction $(\mathrm{EF})$ a few weeks prior at a neighboring hospital.

2.1. Differential Diagnosis. Due to the presentation of a middle age, normotensive black male with heart failure and bradycardia, there was suspicion for an infiltrative cardiomyopathy such as amyloidosis, both light chain and transthyretin types, as well as sarcoidosis with cardiac involvement.

2.2. Investigations. Serial ECGs showed several different bradycardic rhythms (Figure 1). He was noted to have intermittent second-degree Mobitz type 1 atrioventricular block (AVB) but also periods of high-grade AVB. Transthoracic echocardiogram revealed asymmetric left ventricular hypertrophy, normal systolic function (EF estimated at 55\%), normal wall motion, and grade 1 diastolic dysfunction. There was no evidence of outflow tract obstruction, and ventricular filling pressures were estimated to be normal. He underwent 


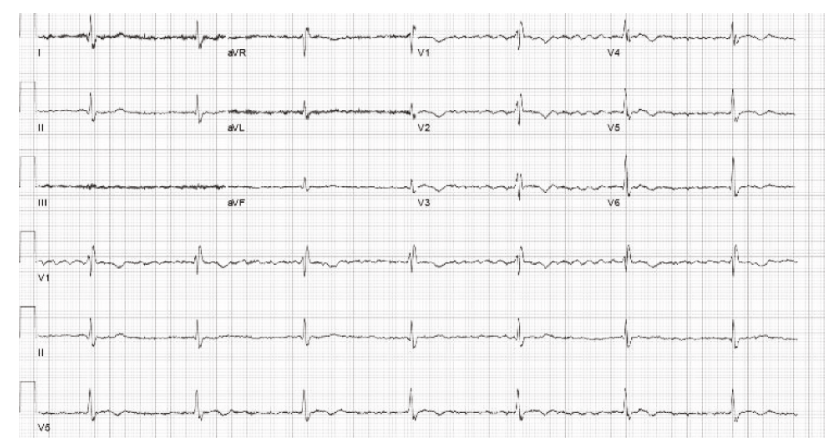

(a)

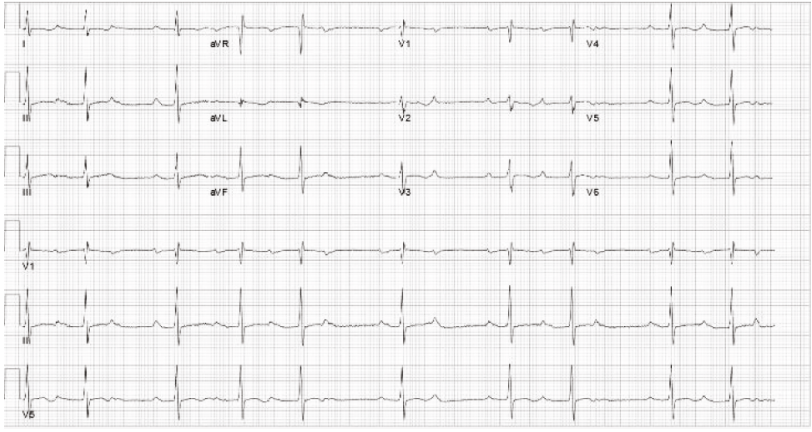

(b)

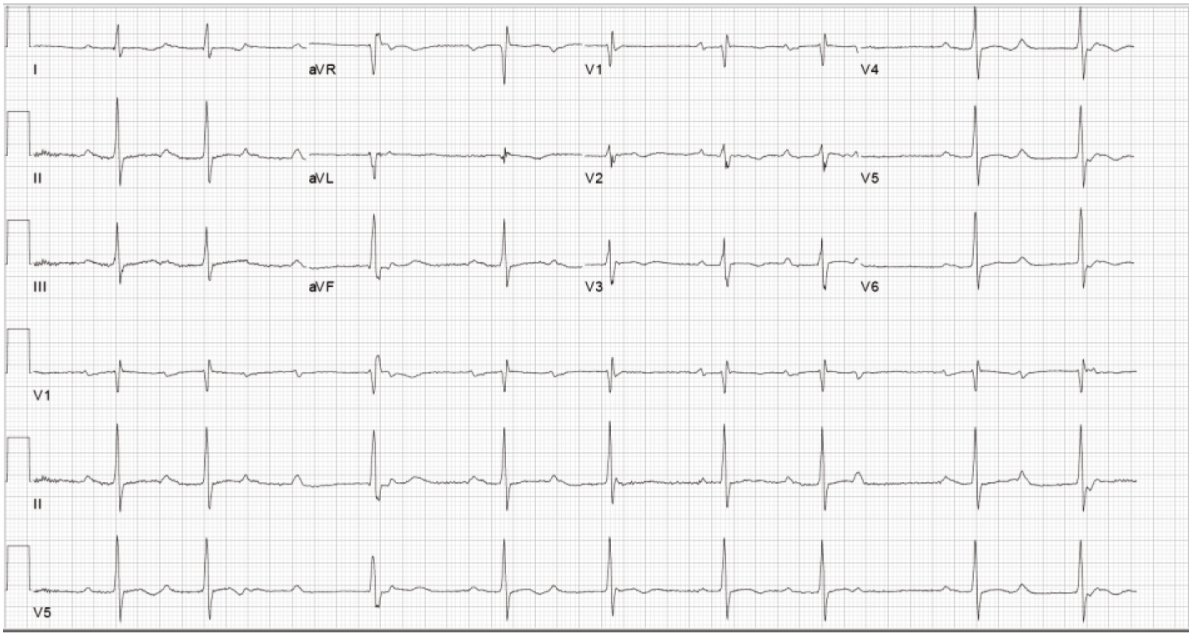

(c)

FIGURE 1: ECG of variable heart block in a patient with cardiac sarcoidosis: (a) baseline atrial fibrillation with junctional escape rhythm; (b) second-degree Mobitz type 1 AV block; (c) high grade AV block.

a treadmill stress test, demonstrating chronotropic competency without evidence of ischemia. Cardiac magnetic resonance (CMR) imaging revealed asymmetric hypertrophy of the ventricular septum with patchy myocardial late gadolinium enhancement involving the mid- to basal septum with heterogeneous uptake in the apex. Labs revealed a normal protein electrophoresis making light chain amyloidosis unlikely. The patient underwent a CT scan of the chest to examine for evidence of pulmonary sarcoidosis; however, this was unremarkable. A technetium-pyrophosphate scan was obtained for assessment of transthyretin amyloid; however, this was negative as well.

The patient was scheduled for follow-up Fluorodeoxyglucose Positron Emission Tomography-Computed Tomography (FDG PET CT) scan. When he obtained the scan, it was positive for FDG uptake in the left ventricular myocardium as well as the apical inferior wall, with no abnormal extracardiac FDG uptake (see Figure 2). These findings were consistent with active isolated cardiac sarcoidosis primarily involving the mid- to basal myocardium.

2.3. Management. During his hospitalization, the patient was administered intravenous loop diuretics for his volume overload. He underwent dual chamber pacemaker implantation, as well as empiric implantable cardiac defibrillator (ICD) placement for primary prevention against ventricular arrhythmias. ICD placement was felt appropriate despite not yet obtaining a definitive diagnosis of cardiac sarcoidosis (CS) for two reasons: (1) the patient's existing need for a pacemaker for treatment of bradycardia and high-grade AVB and (2) the high suspicion for CS. The team did not want to delay primary prevention therapy due to risk of sudden cardiac death in patients with CS and heart block. The patient's cardiac device was set to DDD (dual chamber paced, dual sensed, and dual inhibited) settings given his high degree of block as well as underlying paroxysmal atrial fibrillation. Upon follow-up interrogation of the device, the patient was paced from the right atrial lead $54 \%$ of the time and from the right ventricular lead $100 \%$ of the time and was in atrial fibrillation $28 \%$ of the time, showing his highgrade heart block and underlying bradycardia. He has not had any ventricular arrhythmias to date.

\section{Discussion}

Sarcoidosis is a granulomatous disorder of unknown etiology that can affect multiple organ systems. The histopathologic hallmark of sarcoidosis is the presence of noncaseating granulomas in affected organs. An estimated 5-25\% of patients with sarcoidosis have cardiac involvement [1]. CS can cause 

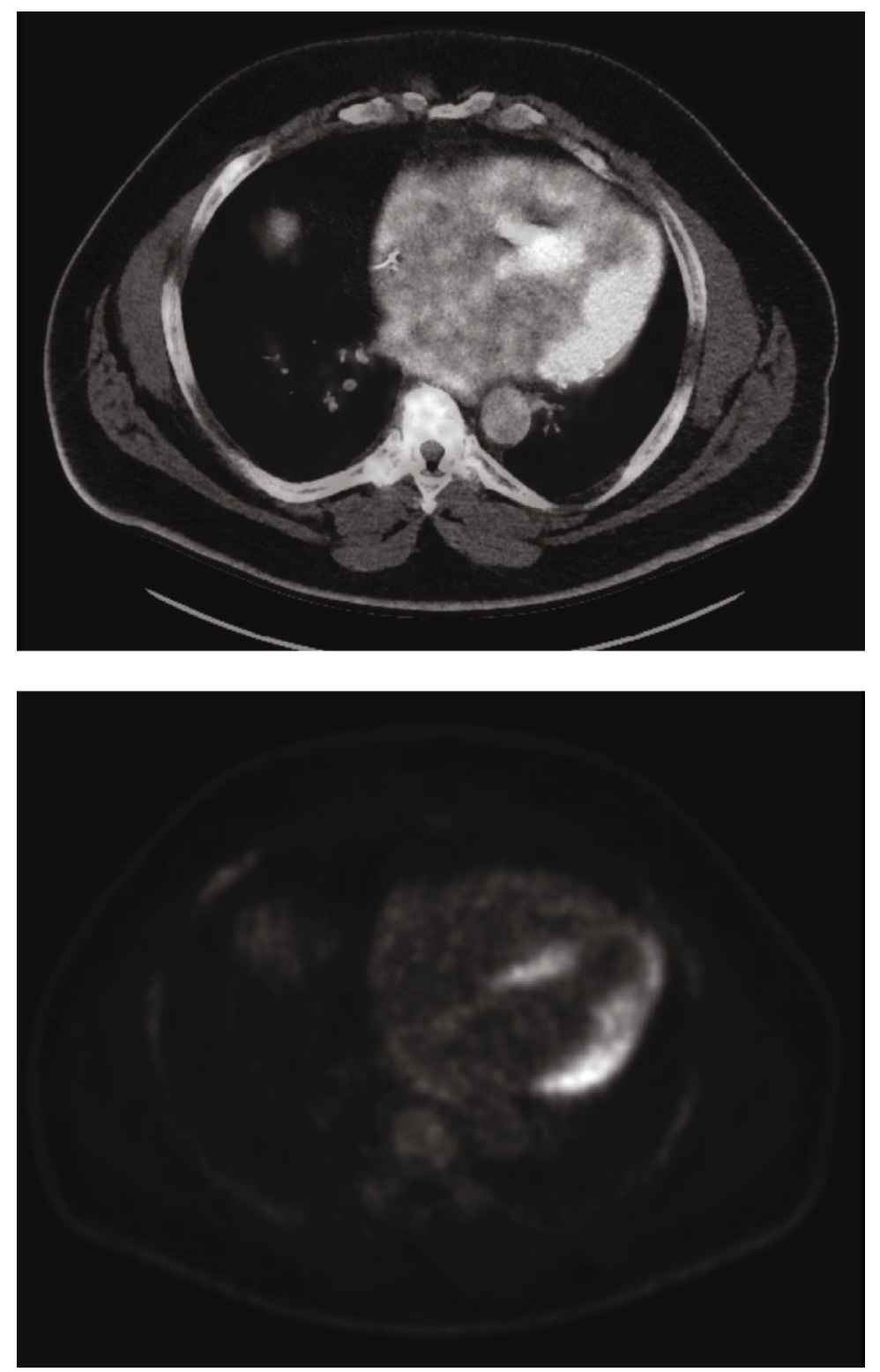

FIGURE 2: Active sarcoid seen in myocardium on FDG PET CT. FDG PET CT showing abnormal uptake in the myocardium as well as apical inferior wall with sparing of the apex with no extracardiac FDR uptake.

a variety of cardiac problems, including heart failure and conduction disease. In a European registry of sarcoidosis patients, high-grade atrioventricular blocks were the most common presentation of those affected, seen in about $44 \%$ of CS patients [2]. Those with CS have increased risk of arrhythmias, particularly ventricular arrhythmias. One cohort study found that the 5-year incidence of VT was $56 \%$ in those with EF below 25\% and 25\% in those with normal EF [2]. There is also an increased risk of sudden cardiac death in CS patients ranging from $9 \%$ in those with normal EF and no history of VT to $34 \%$ in those with EF below $25 \%$ and prior history of VT [2]. Given these risks, diagnosing cardiac sarcoidosis early is imperative.

Previous guidelines for diagnosis required biopsy-proven disease either of the cardiac tissue or extracardiac tissue with other signs of cardiac involvement [3]. These and other European and American diagnostic guidelines have been too reliant on biopsy data, which has poor diagnostic yields, [4] and does not take into account recent advancements in cardiac imaging. New Japanese diagnostic guidelines for CS and isolated cardiac sarcoidosis (ICS) were published in 2017. These new guidelines proposed the diagnosis of CS via major and minor criteria to classify cardiac involvement that includes analysis of ECG, ECHO, CMR, and FDG PET findings (Table 1) [5]. These guidelines have been shown to capture more patients with CS and ICS, allowing them to proceed to recommended therapies such as steroid treatment and cardiac devices for primary prevention [6].

This patient was diagnosed with ICS based on these guidelines as he had FDG-PET data consistent with cardiac 
Table 1: Summary of "New Guidelines for Diagnosis of Cardiac Sarcoidosis in Japan” by Terasaki and Yoshinaga (2017).

(a)

\begin{tabular}{llc}
\hline & Cardiac sarcoidosis & Isolated cardiac sarcoidosis \\
\hline $\begin{array}{l}\text { Histological } \\
\text { diagnosis group }\end{array}$ & $\begin{array}{l}\text { (i) Endomyocardial biopsy or surgical specimens confirm } \\
\text { noncaseating granulomatous tissue }\end{array}$ & $\begin{array}{c}\text { (i) Prerequisite required } \\
\text { (ii) Endomyocardial biopsy or surgical specimens confirm } \\
\text { noncaseating granulomatous tissue }\end{array}$ \\
$\begin{array}{ll}\text { Clinical diagnosis } \\
\text { group }\end{array}$ & $\begin{array}{l}\text { (i) Patient diagnosed with sarcoidosis via tissue biopsy } \\
\text { diagnosis of noncardiac site and }\end{array}$ & $\begin{array}{l}\text { (ii) F-FDG PET reveals abnormally high accumulation of } \\
\text { (ii) Signs of cardiac involvement* }\end{array}$ \\
& $\begin{array}{l}\text { (iii) } 3 / 4 \text { of the other major criteria for cardiac involvement* } \\
\text { are met }\end{array}$ \\
\hline
\end{tabular}

(b)

\begin{tabular}{ll}
\hline & (i) Major criteria \\
& (a) High-grade AV block \\
& (b) Abnormal ventricular wall anatomy \\
* Cardiac involvement & (c) LVEF $<50 \%$ \\
Requires $2 / 5$ major criteria or $1 / 5$ major criteria and & (d) F-FDG PET with increased tracer uptake in cardiac tissue \\
$2 / 3$ minor criteria & (e) Cardiac MRI with delayed gadolinium enhancement in myocardium \\
& (ii) Minor criteria \\
& (a) Abnormal ECG findings \\
& (b) Perfusion defects on myocardial perfusion scintigraphy \\
& (c) Cardiac biopsy with monocyte infiltration or fibrosis \\
& (i) No clinical findings of sarcoidosis in extracardiac organs and no signs of hilar or \\
& (ii) F-FDG PET with no uptake accumulation in extracardiac organs
\end{tabular}

involvement without extracardiac involvement, high-grade AVB, abnormal ventricular wall anatomy, and late gadolinium enhancement seen on CMR.

Treatment with immunosuppression has little high-level data, and most recommendations are driven by expert consensus. One review that discusses induction of steroids for improvement of both ejection fraction and conduction disease described a modest improvement in conduction disease in those treated with steroids, as well as improvement in ejection fraction in those with initial EF below 35\%, however no significant improvement in those with moderately reduced EFs [7]. One systematic review examining the benefits of steroid treatments in CS patients concluded that treatment with exogenous corticosteroids improved conduction disease in about 50\% of those treated; however, there was too little data to support recommendations in steroids to treat ventricular arrhythmia burden or mortality [8].

3.1. Follow-Up. The patient followed up with outpatient cardiology after discharge. He continues on diuretics and rivaroxaban for anticoagulation given his atrial fibrillation. Follow-up ECHO 6 months after initial diagnosis showed reduced ejection fraction at $25 \%$, and the patient was started on guideline-directed medical therapy for heart failure with reduced ejection fraction with betablocker, RAAS inhibition, and Aldactone. He underwent eye exam and thorough pulmonology workup, which was again negative for extracardiac manifestations of sarcoidosis, and the patient just recently was initiated on systemic corticosteroids at $40 \mathrm{mg}$ daily for three months, with tapering dose to $5-15 \mathrm{mg}$ daily thereafter. A repeat echocardiogram will be obtained in 3 months, and repeat cardiac device interrogation to examine for progression or improvement on conduction disease will occur at one-year follow-up.

\section{Conclusion}

This case of suspected CS with variable high-grade conduction blocks highlights the utilization of recently updated diagnostic guidelines for CS. This case illustrates the need to continue to develop progressive diagnostic guidelines in line with available technology. This can lead to more accurate and earlier diagnosis, which allows for more CS patients to benefit from cardiac device and steroid treatment for arrhythmias and primary prevention of sudden cardiac death.

\section{Data Availability}

There is no underlying data to report.

\section{Additional Points}

Learning Objectives. (i) Cardiac sarcoidosis can present as heart failure or conduction disease such as high-degree AVB or ventricular arrhythmias. (ii) New Japan diagnostic guidelines allows for use of advanced cardiac imaging for 
diagnosis of CS and ICS, addressing the problems of poor diagnostic yield with endomyocardial biopsies and taking advantage of new medical technologies. (iii) Patients with CS, particularly those with reduced EF, are at high risk for ventricular arrhythmias and sudden cardiac death. These can be treated with ICD placement and systemic corticosteroids.

\section{Conflicts of Interest}

The author(s) declare(s) that they have no conflicts of interest.

\section{References}

[1] D. H. Birnie, P. B. Nery, A. C. Ha, and R. S. B. Beanlands, "Cardiac sarcoidosis," Journal of the American College of Cardiology, vol. 68, no. 4, pp. 411-421, 2016.

[2] H. K. Nordenswan, J. Lehtonen, K. Ekström et al., "Outcome of cardiac sarcoidosis presenting with high-grade atrioventricular block," Circulation. Arrhythmia and Electrophysiology, vol. 11, no. 8, p. e006145, 2018.

[3] T. Tachibana and K. Iwai TT, Study on the cause of death in patients with sarcoidosis in Japan, Presented at: XII World Congress on Sarcoidosis, Kyoto, Japan, 1991.

[4] M. K. Bennett, N. A. Gilotra, C. Harrington et al., "Evaluation of the role of endomyocardial biopsy in 851 patients with unexplained heart failure from 2000-2009," Circulation: Heart Failure, vol. 6, no. 4, pp. 676-684, 2013.

[5] F. Terasaki and K. Yoshinaga, "New guidelines for diagnosis of cardiac sarcoidosis in Japan," Ann Nucl Cardiol, vol. 3, no. 1, pp. 42-45, 2017.

[6] H. Kawai, M. Sarai, Y. Kato et al., "Diagnosis of isolated cardiac sarcoidosis based on new guidelines," ESC Hear Fail, vol. 7, no. 5, pp. 2662-2671, 2020.

[7] D. H. Birnie, R. Kandolin, P. B. Nery, and M. Kupari, "Cardiac manifestations of sarcoidosis: diagnosis and management," European Heart Journal, vol. 38, no. 35, pp. 2663-2670, 2016.

[8] M. M. Sadek, D. Yung, D. H. Birnie, R. S. Beanlands, and P. B. Nery, "Corticosteroid therapy for cardiac sarcoidosis: a systematic review," The Canadian Journal of Cardiology, vol. 29, no. 9, pp. 1034-1041, 2013. 